\title{
A note on blow-up of solutions for the nonlocal quasilinear parabolic equation with positive initial energy
}

\section{Zhong Bo Fang*, Lu Sun and Changjun Li}

"Correspondence:

fangzb7777@hotmail.com School of Mathematical Sciences, Ocean University of China, Qingdao, 266100, P.R. China

\begin{abstract}
In this short note, we consider a nonlocal quasilinear parabolic equation in a bounded domain with the Neumann-Robin boundary condition. We establish a blow-up result for a certain solution with positive initial energy.
\end{abstract}

\section{Introduction}

We consider the initial boundary value problem for a nonlocal quasilinear parabolic equation

$$
u_{t}=\Delta_{p} u+|u|^{q-1} u-\frac{1}{m(\Omega)} \int_{\Omega}|u|^{q-1} u d x, \quad x \in \Omega, t>0,
$$

with Neumann-Robin boundary and initial conditions

$$
\begin{aligned}
& |\nabla u|^{p-2} \frac{\partial u}{\partial n}=0, \quad x \in \partial \Omega, t>0, \\
& u(x, 0)=u_{0}(x), \quad x \in \Omega,
\end{aligned}
$$

where $\Omega \subset \mathbf{R}^{N}(N \geq 1)$ is a bounded domain with a smooth boundary, $m(\Omega)$ denotes the Lebesgue measure of the domain $\Omega, \Delta_{p} u=\operatorname{div}\left(|\nabla u|^{p-2} \nabla u\right)$ with $p \geq 2, q>p-1, u_{0}(x) \in$ $L^{\infty}(\Omega) \cap W^{1, p}(\Omega), u_{0}(x) \not \equiv 0$, and $\int_{\Omega} u_{0} d x=0$. It is easy to check that the integral of $u$ over $\Omega$ is conserved. Meanwhile, since $u(x, t)$ is not required to be nonnegative, we use $|u|^{q-1} u$ instead of $u^{q}$ in equation (1.1).

Equation (1.1) arises naturally from the fluid mechanics, biology, and population dynamics. In particular, it is a possible model for the diffusion system of some biological species with a human-controlled distribution, in which $u(x, t), \operatorname{div}\left(|\nabla u|^{p-2} \nabla u\right),|u|^{q-1} u$, and $-\frac{1}{m(\Omega)} \int_{\Omega}|u|^{q-1} u d x$ represent the density of the species, the mutation, which we may view as the spread of the characteristic, the growth source of the species, and the humancontrolled distribution at position $x$ and time $t$, respectively. The arising of a nonlocal term denotes the evolution of the species at a point of space, which depends not only on nearby density, but also on the mean value of the total amount of species due to the effects of spatial inhomogeneity, see [1-3]. This equation can be also used to describe the slow diffusion of concentration of non-Newton flow in a porous medium or the temperature of 
some combustible substance ( $c f$. [4-6]). In addition, when $p=q=2$ in (1.1), equation (1.1) becomes

$$
u_{t}=\Delta u+u^{2}-\frac{1}{m(\Omega)} \int_{\Omega} u^{2} d x, \quad x \in \Omega, t>0
$$

which is one of the simplest equations with nonlocal terms and a homogeneous Neumann boundary condition, and the quantity $\int_{\Omega} u(x, t) d x$ is conserved. This equation is also related to the Navier-Stokes equation on an infinite slab, which is explained in [7].

In recent years, blow-up theory for solutions of the initial boundary value problem of parabolic equations with local or nonlocal term has been rapidly developed, and there have been many delicate results. Especially, for the relations between initial energy and blow-up solution, see [8-14]. As for researches on the initial boundary value problem of semilinear parabolic equations, we refer the readers to [8-12]. For instances, Hu and Yin [8] considered the nonlocal semilinear equation

$$
u_{t}=\Delta u+|u|^{q-1} u-\frac{1}{m(\Omega)} \int_{\Omega}|u|^{q-1} u d x, \quad x \in \Omega, t>0
$$

with a homogeneous Neumann boundary condition

$$
\frac{\partial u}{\partial n}=0, \quad x \in \partial \Omega, t>0
$$

and established a result of local existence for the negative initial energy by using a convexity argument. Souf [9] investigated a similar problem and established a relation between the finite time blow-up of solutions and the negativity of initial energy for $1<q \leq 2$ by using a gamma-convergence argument. They also conjectured that the relation might hold for all $q>1$, and a positive answer to which was given by Jazar in [10]. Lately, by using the energy method, Gao [11] established a relation between the finite time blow-up of solutions and the positivity of initial energy of problem (1.4)-(1.5). In addition, Niculescu and Rovența [12] considered a more general initial boundary value problem of nonlocal semilinear parabolic equation given by

$$
u_{t}=\Delta u+f(|u|)-\frac{1}{m(\Omega)} \int_{\Omega} f(|u|) d x, \quad x \in \Omega, t>0,
$$

with homogeneous Neumann boundary condition (1.5), and established a blow-up result, when $f(|u|)$ belongs to a large class of nonlinearities and the initial energy was nonpositive by using the convexity method. For the initial boundary value problem of quasilinear parabolic equations, Liu and Wang [13] studied the local $p$-Laplacian equation

$$
u_{t}=\Delta_{p} u+f(u), \quad x \in \Omega, t>0,
$$

with homogeneous Dirichlet boundary condition, and built a relation between the finite time blow-up of solutions and the positivity of initial energy. Recently, Niculescu and Rovența [14] considered the nonlocal quasilinear equation

$$
u_{t}=\Delta_{p} u+f(|u|)-\frac{1}{m(\Omega)} \int_{\Omega} f(|u|) d x, \quad x \in \Omega, t>0,
$$


with the Neumann-Robin boundary condition (1.2), and established a relation between the finite time blow-up solutions and the negative initial energy, when $p \geq 2$ and $f$ belongs to a large class of nonlinearities by virtue of a convexity argument.

In those works mentioned above, most problems assumed that the initial energy was negative or non-positive to ensure the occurrence of blow-up. But, to the best of our knowledge, the positive initial energy can also ensure the occurrence of blow-up in local or nonlocal problems. It is difficult to determine whether the solutions of the initial boundary value problem of nonlocal equation (1.1) will blow up in finite time, since the comparison principle, which is the most effective tool to show blow-up of solutions, is invalid. The aim of our work is to find a relation between the finite time blow-up of solutions and the positive initial energy of problem (1.1)-(1.3) by the improved convexity method.

\section{Preliminaries and the main result}

Since $p>2$, equation (1.1) is degenerate on $\{(x, t) \mid \nabla u=0\}$, there is no classical solution in general. Hence, it is reasonable to find a weak solution of problem (1.1)-(1.3). To this end, we first give the following definition of the weak solution of problem (1.1)-(1.3).

Definition 1 If a function $u(x, t)$ satisfies the following conditions:

(1) $u \in L^{\infty}\left(Q_{T}\right) \cap L^{p}\left(0, T ; W^{1, p}(\Omega)\right), \quad u_{t} \in L^{2}\left(Q_{T}\right)$

(2) $\iint_{Q_{T}}\left[u \phi_{t}-|\nabla u|^{p-2} \nabla u \cdot \nabla \phi+\left(|u|^{q-1} u-\frac{1}{m(\Omega)} \int_{\Omega}|u|^{q-1} u d x\right) \phi\right] d x d t$

$$
=\int_{\Omega} u(x, t) \phi(x, t) d x-\int_{\Omega} u(x, 0) \phi(x, 0) d x \quad \text { for every } t \in(0, T),
$$

where $\phi \in C^{1}(\bar{\Omega} \times[0, T])$ and $Q_{T}=\Omega \times(0, T)$, then $u(x, t)$ is called a weak solution of problem (1.1)-(1.3).

Remark 1 The existence of local nonnegative solutions in time to problem (1.1)-(1.3) can be obtained by using a fixed point theorem or a parabolic regular theory to get a suitable estimate in a standard limiting process, see $[6,15,16]$. The proof is standard, and so it is omitted here. Moreover, for convenience, we may assume that the appropriate weak solution is smooth, and no longer consider approximation problem.

Let $W(\Omega)$ denote a subspace of $W^{1, p}(\Omega)$, and we assume that the functions $u$ in $W(\Omega)$ satisfy $\int_{\Omega} u d x=0$. We also define a norm on $W(\Omega)$ by

$$
\|u\|=\left(\int_{\Omega}|\nabla u|^{p} d x\right)^{\frac{1}{p}}
$$

It is easy to see that this norm is equivalent to the classical norm on $W^{1, p}(\Omega)$ by using the Poincaré inequality. Set $B$ be the optimal constant of the embedding inequality

$$
\|u\|_{q+1} \leq B\|\nabla u\|_{p}, \quad u \in W(\Omega),
$$


which is equivalent to

$$
B^{-1}=\inf _{u \in W(\Omega), u \neq 0} \frac{\|\nabla u\|_{p}}{\|u\|_{q+1}},
$$

where

$$
1<q \leq+\infty, \quad \text { when } N \leq p ; \quad 1<q \leq \frac{(p-1) N+p}{N-p}, \quad \text { when } N>p .
$$

We also define $\alpha_{1}, E_{1}$, and $E(t)$ as

$$
\alpha_{1}=B^{-\frac{q+1}{q-p+1}}, \quad E_{1}=\left(\frac{1}{p}-\frac{1}{q+1}\right) B^{-\frac{p(q+1)}{q-p+1}} \quad \text { for } q>p-1
$$

and

$$
E(t)=\int_{\Omega}\left[\frac{1}{p}|\nabla u|^{p}-\frac{1}{q+1}|u|^{q+1}\right] d x .
$$

We now introduce our main result on the blow-up solutions with the positive initial energy below.

Theorem 1 (Sufficient condition for blow-up) Set $p \geq 2, p-1<q \leq+\infty$, when $N \leq p$ and $p-1<q \leq \frac{(p-1) N+p}{N-p}$, when $N>p$. Suppose that $u(\cdot, t) \in W(\Omega)$ is a solution of $(1.1)-(1.3)$, and the initial datum $u_{0}(x) \in W(\Omega)$ is chosen to ensure that $E(0)<E_{1}$ and $\left\|\nabla u_{0}\right\|_{p}>\alpha_{1}$. Then the solution $u(x, t)$ blows up in a finite time.

Remark 2 Choose $\Omega=\left(-\frac{\pi}{2}, \frac{\pi}{2}\right), p=3$ and $q=3$; one can easily verify that $u_{0}(x)=\sin x$ satisfies $u_{0}(x) \in W(\Omega), E(0)<E_{1}$ and $\left\|\nabla u_{0}\right\|_{p}>\alpha_{1}$, therefore, conditions in Theorem 1 are valid.

Remark 3 Our result improves the results of Gao [11] and Niculescu and Rovența [14].

\section{The proof of Theorem 1}

To prove our main result, we first establish the following three lemmas obtained by applying the idea of Liu and Wang in [13], where a different type of problem was discussed.

Lemma $1 E(t)$ defined in (2.3) is non-increasing in $t$.

Proof A direct computation with the integration by parts yields

$$
\begin{aligned}
\frac{d}{d t} E(t) & =-\int_{\Omega} u_{t}\left(\Delta_{p} u+|u|^{q-1} u\right) d x \\
& =-\int_{\Omega} u_{t}^{2} d x-\frac{1}{m(\Omega)} \int_{\Omega}|u|^{q-1} u d x \cdot \int_{\Omega} u_{t} d x=-\int_{\Omega} u_{t}^{2} d x \leq 0,
\end{aligned}
$$

and hence, $E(t)$ is non-increasing in $t$.

The following second lemma gives a lower bound estimate for the solution $u(x, t)$ in the $L^{p}$-norm: 
Lemma 2 Let $u(x, t)$ be a solution of (1.1)-(1.3) with initial data satisfying

$$
E(0)<E_{1} \text { and }\left\|\nabla u_{0}\right\|_{p}>\alpha_{1} .
$$

Then there exists a positive constant $\alpha_{2}>\alpha_{1}$ such that

$$
\|\nabla u\|_{p}>\alpha_{2}, \quad \forall t \geq 0
$$

and

$$
\|u\|_{q+1} \geq B \alpha_{2}, \quad \forall t \geq 0 .
$$

Proof By (2.1) and (2.3), we notice that

$$
\begin{aligned}
E(t) & \geq \frac{1}{p}\|\nabla u\|_{p}^{p}-\frac{1}{q+1} B^{q+1}\|\nabla u\|_{p}^{q+1} \\
& =\frac{1}{p} \alpha^{p}-\frac{1}{q+1} B^{q+1} \alpha^{q+1} \doteq g(\alpha),
\end{aligned}
$$

where $\alpha=\|\nabla u\|_{p}$. It can be easily seen that $g$ is increasing for $0<\alpha<\alpha_{1}$, and decreasing for $\alpha>\alpha_{1}, g(\alpha) \rightarrow-\infty$ as $\alpha \rightarrow+\infty$, and $g\left(\alpha_{1}\right)=E_{1}$, where $\alpha_{1}$ and $E_{1}$ are constants defined in (2.2). Therefore, there exists a constant $\alpha_{2}>\alpha_{1}$ such that $E(0)=g\left(\alpha_{2}\right)$, since $E(0)<E_{1}$.

Setting $\alpha_{0}=\left\|\nabla u_{0}\right\|_{p}$, we have $g\left(\alpha_{0}\right) \leq E(0)=g\left(\alpha_{2}\right)$ by (3.3), which implies that $\alpha_{0} \geq \alpha_{2}$, since $\alpha_{0}$ and $\alpha_{2} \geq \alpha_{1}$.

To establish (3.1), we assume that there exists a constant $t_{0}>0$ such that $\left\|\nabla u\left(\cdot, t_{0}\right)\right\|_{p}<\alpha_{2}$. Because of the continuity of $\|\nabla u(\cdot, t)\|_{p}$, we can choose $t_{0}$ such that $\left\|\nabla u\left(\cdot, t_{0}\right)\right\|_{p}>\alpha_{1}$. From (3.3), we deduce that

$$
E(0)=g\left(\alpha_{2}\right)<g\left(\left\|\nabla u\left(\cdot, t_{0}\right)\right\|_{p}\right) \leq E\left(t_{0}\right),
$$

which is impossible by Lemma 1 , and hence, inequality (3.1) is established.

It also follows from (2.3) that

$$
\frac{1}{p}\|\nabla u\|_{p}^{p} \leq E(0)+\frac{1}{q+1} \int_{\Omega}|u|^{q+1} d x .
$$

We then obtain that

$$
\begin{aligned}
\frac{1}{q+1} \int_{\Omega}|u|^{q+1} d x & \geq \frac{1}{p}\|\nabla u\|_{p}^{p}-E(0) \\
& \geq \frac{1}{p} \alpha_{2}^{p}-E(0)=\frac{1}{p} \alpha_{2}^{p}-g\left(\alpha_{2}\right) \\
& =\frac{1}{q+1} B^{q+1} \alpha_{2}^{q+1},
\end{aligned}
$$

from which inequality (3.2) follows. 
Setting

$$
H(t)=E_{1}-E(t), \quad \forall t \geq 0,
$$

we have the following lemma.

Lemma 3 For all $t \geq 0$, we have the inequalities

$$
0<H(0) \leq H(t) \leq \frac{1}{q+1} \int_{\Omega}|u|^{q+1} d x .
$$

Proof By Lemma 1, we have

$$
H^{\prime}(t)=-E^{\prime}(t) \geq 0,
$$

and so

$$
H(t) \geq H(0)>0, \quad t \geq 0 .
$$

From (2.3) and (3.4), we get

$$
H(t)=E_{1}-\frac{1}{p}\|\nabla u\|_{p}^{p}+\frac{1}{q+1} \int_{\Omega}|u|^{q+1} d x .
$$

It then follows from (3.1) and (3.3) that

$$
E_{1}-\frac{1}{p}\|\nabla u\|_{p}^{p} \leq E_{1}-\frac{1}{p} \alpha_{2}^{p} \leq-\frac{1}{q+1} B^{q+1} \alpha_{1}^{q+1} \leq 0, \quad t \geq 0
$$

which guarantees (3.5).

Proof of Theorem 1 Setting $G(t)=\frac{1}{2} \int_{\Omega} u^{2}(x, t) d x$ and differentiating it, we obtain that

$$
\begin{aligned}
G^{\prime}(t) & =\int_{\Omega} u u_{t} d x \\
& =\int_{\Omega} u\left(\Delta_{p} u+|u|^{q-1} u-\frac{1}{m(\Omega)} \int_{\Omega}|u|^{q-1} u d x\right) d x \\
& =\int_{\Omega}|u|^{q+1} d x-\int_{\Omega}|\nabla u|^{p} d x \\
& =\int_{\Omega}|u|^{q+1} d x-p E(t)-\frac{p}{q+1} \int_{\Omega}|u|^{q+1} d x \\
& =\frac{q-p+1}{q+1} \int_{\Omega}|u|^{q+1} d x-p E_{1}+p H(t) .
\end{aligned}
$$

From (2.2) and (3.2), we deduce that

$$
\begin{aligned}
p E_{1} & =p\left(\frac{1}{p}-\frac{1}{q+1}\right) B^{-\frac{p(q+1)}{q-p+1}} \\
& =\frac{q-p+1}{q+1} \frac{\alpha_{1}^{q+1}}{\alpha_{2}^{q+1}} B^{q+1} \alpha_{2}^{q+1} \leq \frac{q-p+1}{q+1} \frac{\alpha_{1}^{q+1}}{\alpha_{2}^{q+1}} \int_{\Omega}|u|^{q+1} d x .
\end{aligned}
$$


Substituting (3.7) into (3.6), we obtain

$$
\begin{aligned}
G^{\prime}(t) & \geq\left(1-\frac{\alpha_{1}^{q+1}}{\alpha_{2}^{q+1}}\right) \frac{q-p+1}{q+1} \int_{\Omega}|u|^{q+1} d x+p H(t) \\
& =C_{0} \int_{\Omega}|u|^{q+1} d x+p H(t)
\end{aligned}
$$

where $C_{0}=\left(1-\frac{\alpha_{1}^{q+1}}{\alpha_{2}^{q+1}}\right) \frac{q-p+1}{q+1}$.

By Hölder's inequality, we get

$$
G^{\frac{q+1}{2}}(t)=\left(\frac{1}{2} \int_{\Omega}|u|^{2}(x, t) d x\right)^{\frac{q+1}{2}} \leq C \int_{\Omega}|u|^{q+1} d x,
$$

where $C=C(|\Omega|, q)>0$. Combining (3.8) and (3.9) with Lemma 3, we have

$$
G^{\prime}(t) \geq \gamma G^{\frac{q+1}{2}}(t), \quad \text { where } \gamma=\frac{C_{0}}{C}>0 .
$$

Integrating (3.10) over $(0, t)$, we obtain

$$
G^{\frac{q-1}{2}}(t) \geq \frac{1}{G^{\frac{1-q}{2}}(0)-\frac{q-1}{2} \gamma t},
$$

which implies that $G(t)$ blows up at a finite time $T^{*} \leq \frac{G^{\frac{1-q}{2}}(0)}{\frac{q-1}{2} \gamma}$, and so does $u(x, t)$. The proof is completed.

Remark 4 Due to the restriction of our method, we cannot get the blow-up result for $q>\frac{(p-1) N+p}{N-p}$, when $N>p$. We conjecture that Theorem 1 will hold for all $q>p-1 \geq 1$.

\section{Competing interests}

The authors declare that they have no competing interests.

\section{Authors' contributions}

All authors contributed equally to the manuscript and read and approved the final manuscript.

\section{Acknowledgements}

This work is supported by the Natural Science Foundation of Shandong Province of China (ZR2012AM018). The authors would like to deeply thank all the reviewers for their insightful and constructive comments.

Received: 2 March 2013 Accepted: 23 July 2013 Published: 8 August 2013

\section{References}

1. Furter, J, Grinfield, M: Local vs. non-local interactions in populations dynamics. J. Math. Biol. 27, 65-80 (1989)

2. Calsina, A, Perello, C, Saldana, J: Non-local reaction-diffusion equations modelling predator-prey coevolution. Publ. Mat. 38, 315-325 (1994)

3. Allegretto, W, Fragnelli, G, Nistri, P, et al.: Coexistence and optimal control problems for a degenerate predator-prey model. J. Math. Anal. Appl. 378, 528-540 (2011)

4. Bebernes, J, Eberly, D: Mathematical Problems from Combustion Theory. Springer, New York (1989)

5. Pao, CV: Nonlinear Parabolic and Elliptic Equations. Plenum, New York (1992)

6. Wu, ZQ, Zhao, JN, Yin, JX, et al.: Nonlinear Diffusion Equations. World Scientific, Singapore (2001)

7. Budd, CJ, Dold, JW, Stuart, AM: Blow-up in a system of partial differential equations with conserved first integral. Part II: problems with convection. SIAM J. Appl. Math. 54(3), 610-640 (1994)

8. Hu, B, Yin, HM: Semi-linear parabolic equations with prescribed energy. Rend. Circ. Mat. Palermo 44, 479-505 (1995) 
9. El Soufi, A, Jazar, M, Monneau, R: A gamma-convergence argument for the blow-up of a non-local semilinear parabolic equation with Neumann boundary conditions. Ann. Inst. Henri Poincaré, Anal. Non Linéaire 24(1), 17-39 (1995)

10. Jazar, M, Kiwan, R: Blow-up of a non-local semilinear parabolic equation with Neumann boundary conditions. Ann. Inst. Henri Poincaré, Anal. Non Linéaire 25, 215-218 (2008)

11. Gao, WJ, Han, YZ: Blow-up of a nonlocal semilinear parabolic equation with positive initial energy. Appl. Math. Lett. 24(5), 784-788 (2011)

12. Niculescu, CP, Rovenţa, J: Large solutions for semilinear parabolic equations involving some special classes of nonlinearities. Discrete Dyn. Nat. Soc. 2010, Article ID 491023 (2010)

13. Liu, WJ, Wang, MX: Blow-up of the solution for a $p$-Laplacian equation with positive initial energy. Acta Appl. Math. $103,141-146(2008)$

14. Niculescu, CP, Rovenţa, J: Generalized convexity and the existence of finite time blow-up solutions for an evolutionary problem. Nonlinear Anal. TMA 75, 270-277 (2012)

15. Zhao, JN: Existence and nonexistence of solutions for $u_{t}=\operatorname{div}\left(|\nabla u|^{p-2} \nabla u\right)+f(\nabla u, u, x, t)$. J. Math. Anal. Appl. 172, 130-146 (1993)

16. Li, FC, Xie, CH: Global and blow-up solutions to a p-Laplace equation with nonlocal source. Comput. Math. Appl. 46, 1525-1533 (2003)

doi:10.1186/1687-2770-2013-181

Cite this article as: Fang et al.: A note on blow-up of solutions for the nonlocal quasilinear parabolic equation with positive initial energy. Boundary Value Problems 2013 2013:181.

\section{Submit your manuscript to a SpringerOpen ${ }^{\circ}$ journal and benefit from:}

- Convenient online submission

- Rigorous peer review

- Immediate publication on acceptance

Open access: articles freely available online

- High visibility within the field

- Retaining the copyright to your article 